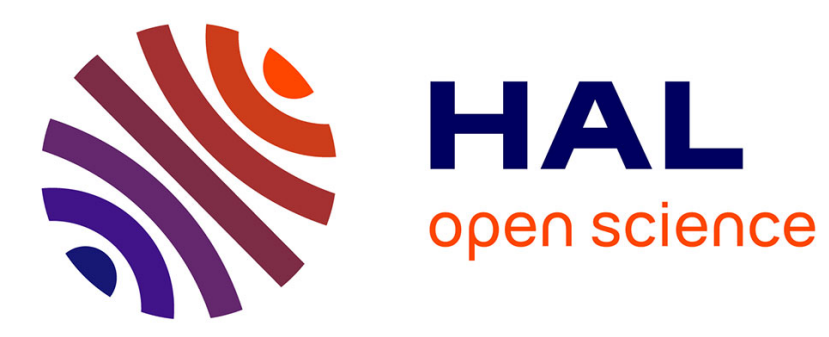

\title{
Recent progress on univariate and multivariate polynomial and spline quasi-interpolants
}

\author{
Paul Sablonnière
}

\section{To cite this version:}

Paul Sablonnière. Recent progress on univariate and multivariate polynomial and spline quasiinterpolants. Detlef H. Mache, József Szabados and Marcel G. de Bruin. Trends and applications in constructive approximation. Proceedings of the 4th IBoMAT Conference held in Witten Bommerholz, 2004, Springer, pp.229-245, 2005, International Series of Numerical Mathematics. 151, 3-7643-7124-2. 10.1007/3-7643-7356-3_17. hal-00001512

\section{HAL Id: hal-00001512 https://hal.science/hal-00001512}

Submitted on 3 May 2004

HAL is a multi-disciplinary open access archive for the deposit and dissemination of scientific research documents, whether they are published or not. The documents may come from teaching and research institutions in France or abroad, or from public or private research centers.
L'archive ouverte pluridisciplinaire HAL, est destinée au dépôt et à la diffusion de documents scientifiques de niveau recherche, publiés ou non, émanant des établissements d'enseignement et de recherche français ou étrangers, des laboratoires publics ou privés. 


\title{
Recent progress on univariate and multivariate polynomial and spline quasi-interpolants
}

\author{
Paul Sablonnière
}

\begin{abstract}
Polynomial and spline quasi-interpolants (QIs) are practical and effective approximation operators. Among their remarkable properties, let us cite for example: good shape properties, easy computation and evaluation (no linear system to solve), uniform boundedness independently of the degree (polynomials) or of the partition (splines), good approximation order. We shall emphasize new results on various types of univariate and multivariate polynomial or spline QIs, depending on the nature of coefficient functionals, which can be differential, discrete or integral. We shall also present some applications of QIs to numerical methods.
\end{abstract}

\section{Introduction}

A quasi-interpolant of $f$ has the general form

$$
Q f=\sum_{\alpha \in A} \mu_{\alpha}(f) B_{\alpha}
$$

where $\left\{B_{\alpha}, \alpha \in A\right\}$ is a family of polynomials or B-splines forming a partition of unity, and $\left\{\mu_{\alpha}(f), \alpha \in A\right\}$ is a family of linear functionals which are local in the sense that they only use values of $f$ in some neighbourhood of $\Sigma_{\alpha}=\operatorname{supp}\left(B_{\alpha}\right)$. The main interest of QIs is that they provide excellent approximants of functions without solving any linear system of equations. In the literature, one can find the three following types of QIs:

(i) Differential QIs (abbr. DQIs) : the linear functionals are linear combinations of values of derivatives of $f$ at some point in $\Sigma_{\alpha}$. 
(ii) Discrete QIs (abbr. dQIs) : the linear functionals are linear combinations of values of $f$ at some points in the neighbourhood of $\Sigma_{\alpha}$.

(iii) Integral QIs (abbr. iQIs) : the linear functionals are linear combinations of weighted mean values of $f$ in the neighbourhood of $\Sigma_{\alpha}$.

We shall present various types of univariate and multivariate polynomial and spline QIs, mainly dQIs and iQIs, which were recently introduced in the literature. For polynomial QIs, we only present QIs which are close to the orifginal Bernstein or Durrmeyer operators (for other types of QIs, see for example [35][36]).

The prototype of polynomial $d Q I s$ is the classical Bernstein operator

$$
B_{n} f=\sum_{i=0}^{n} f\left(\frac{i}{n}\right) b_{i}^{(n)}
$$

where $\left\{b_{i}^{(n)}(x)=C_{n}^{i} x^{i}(1-x)^{n-i}, 0 \leq i \leq n\right\}$ is the Bernstein basis of the space $\mathbb{P}_{n}$ of polynomials of degree at most $n$ (the $C_{n}^{i}$ are binomial coefficients).

The prototype of polynomial iQIs is the Durrmeyer operator [33]

$$
M_{n} f=\sum_{i=0}^{n}\left\langle f, \tilde{b}_{i}^{(n)}\right\rangle b_{i}^{(n)}
$$

where $\tilde{b}_{i}^{(n)}=b_{i}^{(n)} / \int_{0}^{1} b_{i}^{(n)}=(n+1) b_{i}^{(n)}$ and $\langle f, g\rangle=\int_{0}^{1} f g$. Both can be extended to the multivariate case, either on the hypercube or on the simplex. Another extension consists in adding a Jacobi weight in the scalar product.

The prototypes of spline DQIs are de Boor-Fix QIs [11] and their various univariate and multivariate extensions

$$
Q f=\sum_{j \in J} \lambda_{j}(f) B_{j}
$$

Here $\left\{B_{j} j \in J\right\}$ is a family of univariate B-splines of degree $m$ on a nonuniform sequence of knots $\left\{t_{k}\right\}$. Assuming that $\Sigma_{j}=\operatorname{supp}\left(B_{j}\right)=\left[t_{j-m}, t_{j+1}\right]$, we set $E_{m}=\{-m+1, \ldots, 0\}$ and we define $\psi_{j}(t)=\prod_{r \in E_{m}}\left(t_{j+r}-t\right) \in \mathbb{P}_{m}$ for all $j \in J$. For any $\tau \in \Sigma_{j}$, the coefficient functionals are

$$
\lambda_{j}(f)=\frac{1}{(m-1) !} \sum_{l=0}^{m-1}(-1)^{m-l-1} D^{m-l-1} \psi_{j}(\tau) D^{l} f(\tau) .
$$

The prototypes of spline dQIs are the various univariate and multivariate extensions of Schoenberg-Marsden operators [52][53].

$$
S f=\sum_{j \in J} f\left(\tau_{j}\right) B_{j}
$$

where $\tau_{j}$ is an interior point of $\Sigma_{j}=\operatorname{supp}\left(B_{\alpha}\right)$. 
The prototypes of spline iQIs are the various univariate and multivariate extensions of operators [21][63]

$$
T f=\sum_{j \in J}\left\langle f, M_{j}\right\rangle B_{j}
$$

where $M_{j}$ is a B-spline (which can be different from $B_{j}$ ) normalized by $\int_{\Sigma_{j}} M_{j}=1$.

As emphasized by de Boor ([10], chapter XII), a spline QI defined on non uniform partitions has to be uniformly bounded independently of the partition (abbr. UB) in order to be interesting for applications. Therefore, with some coworkers, we have defined various families of QIs satisfying this property and having an infinite norm as small as possible. In general it is difficult to minimize the true norm of the operator, however, it is often possible to minimize an upper bound of this norm: this gives rise to what we have called near-best (abbr. NB) QIs (see [1],[2]-[4], [40]). Numerical applications are still not very much developed. However, QIs can be useful in approximation and estimation [21][22][85], in numerical quadrature [23][73][75] and for the numerical solution of integral or partial differential equations.

\section{Univariate polynomial QIs}

\subsection{Basic operators}

1) The Bernstein-Stancu $Q I[83]$ is defined for $x \in[0,1]$ by

$$
S_{n}^{(\alpha)} f(x)=\sum_{i=0}^{n} f\left(\frac{i}{n}\right) b_{i}^{(n)}(x, \alpha)
$$

where the Bernstein-Stancu basis is defined by $b_{k}^{(n)}(x, \alpha)=C_{n}^{k} \frac{(x)_{\alpha}^{k}(1-x)_{\alpha}^{n-k}}{(1)_{\alpha}^{n}}$.

Here $(x)_{\alpha}^{k}=x(x+\alpha) \ldots(x+(k-1) \alpha)$, for $\alpha \in \mathbb{R}$. For $\alpha=0$, we recover the classical Bernstein basis.

2) The Bernstein- Phillips (or q-Bernstein) $Q I$ ([56]-[59]) is defined for $x \in[0,1]$ by

$$
B_{n}^{q} f(x)=\sum_{i=0}^{n} f\left(\frac{[i]}{[n]}\right) b_{k}^{(n)}(x, q)
$$

where the Bernstein-Phillips or $q$-Bernstein basis is defined for $q \neq 1$ by $b_{k}^{(n)}(x, q)=\Gamma_{n}^{k} x^{k}(1-x)_{q}^{n-k}$. Here $[i]=\frac{1-q^{i}}{1-q},[i] !=\prod_{s=1}^{i}[s], \Gamma_{n}^{k}=\frac{[n] !}{[k] ![n-k] !}$ and $(x)_{q}^{k}=\prod_{s=0}^{k-1}\left(1-q^{s} x\right)$. For $q=1$, we recover the classical Bernstein basis.

Using the notation $e_{s}(x)=x^{s}$ for monomials, it is easy to prove that all the above QIs $\mathcal{B}_{n}$ are exact on $\mathbb{P}_{1}$, i.e. $\mathcal{B}_{n} e_{s}=e_{s}$ for $s=0,1$. Moreover they are degree 
preserving since $\mathcal{B}_{n} e_{s}(x)=e_{s}(x)+r_{s-1}(x, n)$ where $r_{s-1}(x, n)$ is some polynomial of degree at most $s-1$ depending on $n$ (and eventually on the parameters $\alpha, N$ or $q$ ).

QIs-

\subsection{Left and right BQIs}

All operators $\mathcal{B}_{n}$ defined above are isomorphisms of $\mathbb{P}_{n}$. Moreover $\mathcal{B}_{n}$ and $\mathcal{A}_{n}=$ $\mathcal{B}_{n}^{-1}$ can be expressed as linear differential operators with polynomial coefficients

$$
\mathcal{B}_{n}=\sum_{k=0}^{n} \beta_{k}^{(n)} D^{k}, \quad \mathcal{A}_{n}=\sum_{k=0}^{n} \alpha_{k}^{(n)} D^{k}
$$

where $D=\frac{d}{d x}$ and the polynomials $\beta_{k}^{(n)} \in \mathbb{P}_{k}$ and $\alpha_{k}^{(n)} \in \mathbb{P}_{k}$ are defined by simple recursions (see e.g. [64]-[67] for partial results in this sense).

For $0 \leq r \leq n$, we introduce the partial inverses:

$$
\mathcal{A}_{n}^{(r)}=\sum_{k=0}^{r} \alpha_{k}^{(n)} D^{k}
$$

and we consider the two families of right and left BQIs:

(RBQI) The right BQIs $\mathcal{B}_{n}^{[r]}=\mathcal{B}_{n} \circ \mathcal{A}_{n}^{(r)}$ are defined for $C^{r}$-functions $f$ by

$$
\mathcal{B}_{n}^{[r]} f=\mathcal{B}_{n}\left(\mathcal{A}_{n}^{(r)} f\right)=\mathcal{B}_{n}\left(\sum_{k=0}^{r} \alpha_{k}^{(n)} D^{k} f\right) .
$$

(LBQI) The left BQIs $\mathcal{B}_{n}^{(r)}=\mathcal{A}_{n}^{(r)} \circ \mathcal{B}_{n}$ are defined on any (e.g. continuous) function

$$
\mathcal{B}_{n}^{(r)} f=\mathcal{A}_{n}^{(r)}\left(\mathcal{B}_{n} f\right)=\sum_{k=0}^{r} \alpha_{k}^{(n)} D^{k}\left(\mathcal{B}_{n} f\right)
$$

By construction, for $0 \leq r \leq n$, the BQIs $\mathcal{B}_{n}^{[r]}$ and $\mathcal{B}_{n}^{(r)}$ are exact on the space $\mathbb{P}_{r}$. Moreover, in many cases, the LBQIs have a uniformly bounded infinite norm, independent on $n$ for each $0 \leq k \leq n$ fixed (see e.g. [70] [86] for some results of this type). From this property are deduced some convergence results (see [30][67]).

\subsection{Kageyama QIs}

Kageyama [44] [45] considers Stancu operators for $\alpha \in\left[-\frac{1}{n}, 0\right]$

$$
S_{n}^{\left(-\frac{1}{n}\right)}=\mathcal{L}_{n}, \quad S_{n}^{(0)}=B_{n},
$$


where $\mathcal{L}_{n}$ is the Lagrange interpolation operator on the uniform partition of $[0,1]$ (this result is due to Mühlbach). Then he truncates at order $s$ the Maclaurin series of $S_{n}^{(\alpha)} f$ w.r.t. $\alpha$ and he takes the value of this polynomial at $\alpha=-\frac{1}{n}$ :

$$
\mathcal{K}_{n}^{(s)} f=\sum_{j=0}^{s} \frac{1}{j !} \frac{(-1)^{j}}{n^{j}} \frac{\partial^{j}}{\partial \alpha^{j}}\left[S_{n}^{(\alpha)} f\right]_{\alpha=0}
$$

$\mathcal{K}_{n}^{(0)}=S_{n}^{(0)}=B_{n}$ and $\mathcal{K}_{n}^{(\infty)}=S_{n}^{\left(-\frac{1}{n}\right)}=\mathcal{L}_{n}$. He also gives expansions of $\mathcal{K}_{n} f$ in terms of derivatives of $B_{n} f$ and in powers of $\frac{1}{n}$. He proves that, for all $s$ fixed, $\left\|\mathcal{K}_{n}^{(s)}\right\|_{\infty}$ is uniformly bounded and give Voronovskaja type results, e.g.

$$
\lim _{n \rightarrow \infty} n^{s+1}\left(\mathcal{K}_{n}^{(s)} f-f\right)=-\sum_{k=0}^{2 s+2} \frac{1}{k !} \Upsilon_{s+1, k} D^{k} f
$$

where the polynomials $\Upsilon_{s+1, k}$ can be computed by recursion. He also compares the expansions of $\mathcal{L}_{n}$, the BQIs $B_{n}^{(r)}$ and $\mathcal{K}_{n}^{(s)}$ interms of derivatives of $B_{n} f$ with polynomial coefficients. Numerical experiments done by the author suggest that these operators are in general better approximants than BQIs of section 3.1.

\subsection{Univariate Durrmeyer and Goodman-Sharma QIs}

A straightforward generalization of the Durrmeyer operator $M_{n}$ consists in introducing a Jacobi weight on $[0,1]$ in the associated scalar product

$$
\langle f, g\rangle=\int_{0}^{1} w_{\alpha, \beta}(t) f(t) g(t) d t, \quad w_{\alpha, \beta}(t)=t^{\alpha}(1-t)^{\beta}, \text { for } \alpha, \beta>-1
$$

The extended Durrmeyer-Jacobi operator $([6],[61])$ is then defined by

$$
M_{n}^{(\alpha, \beta)} f=\sum_{i=0}^{n} \frac{\left\langle f, b_{i}^{(n)}\right\rangle}{\left\langle e_{0}, b_{i}^{(n)} \cdot\right\rangle} b_{i}^{(n)}
$$

The limit case $(\alpha, \beta)=(-1,-1)$, corresponding to the weight $\tilde{w}(x)=\frac{1}{x(1-x)}$, gives a QI with very attractive properties. It has been introduced by Goodman and Sharma [38][39] for polynomial (and a variant for spline) QIs. It can be written as follows, with $L f(x)=(1-x) f(0)+x f(1)$ :

$$
G_{n} f=L f+(n-1) \sum_{i=1}^{n-1}\left\langle f-L f, b_{i-1}^{(n-2)}\right\rangle b_{i}^{(n)} .
$$

This operator is exact on $\mathbb{P}_{1}$ and its behaviour is quite similar to that of the classical Bernstein operator. For example, one has for $f \in C^{2}(I)$

$$
\lim n\left(f(x)-G_{n} f(x)\right)=x(1-x) f^{\prime \prime}(x) .
$$


It also preserves the positivity, the monotonicity and the convexity of $f$. As discrete Bernstein operators, the above operators $G_{n}$ have associated QIs in the sense of section $3.1[70]$.

\subsection{Extrapolation}

All operators $\mathcal{B}_{n}$ described in this section have asymptotic expansions of type

$$
\mathcal{B}_{n} f(x) \sim f(x)+\sum_{k \geq r} \frac{\varphi_{k}^{(n)}(f, x)}{n^{k}}
$$

for some index $r$, the $\varphi_{k}^{(n)}(f, x)$ being linear differential operators depending on $n$ and $k$. Therefore they are good candidates for extrapolation methods (see e.g. [14] and [82]). Numerical experiments done by the author show that Richardson extrapolation is efficient while the use of variants of epsilon or $\Delta^{2}$ algorithms often introduce spurious poles in the interval of definition.

\section{Polynomial QIs on a simplex}

\subsection{Bernstein operator and associated QIs}

The simplex $S$ of dimension $d-1$ is defined in barycentric coordinates as $S=\left\{\mathbf{x}=\left(x_{1}, x_{2}, \ldots, x_{d}\right):|\mathbf{x}|=1\right\}$ with $|\mathbf{x}|=\sum_{i=1}^{d}\left|x_{i}\right|$.

The associated simplex of indices, monomials and partial derivatives are defined by $\Sigma_{n}=\left\{\mathbf{i}=\left(i_{1}, i_{2}, \ldots, i_{d}\right):|\mathbf{i}|=n\right\}, X_{n}=\left\{\frac{\mathbf{i}}{n}: \mathbf{i} \in \Sigma_{n}\right\} \subset S$,

$\mathbf{i} !=i_{1} ! i_{2} ! \ldots, i_{d} !, \quad \mathbf{x}^{\mathbf{i}}=x_{1}^{i_{1}} x_{2}^{i_{2}} \ldots x_{d}^{i_{d}}, D^{\mathbf{i}}=D_{1}^{i_{1}} D_{2}^{i_{2}} \ldots D_{d}^{i_{d}}$ with $D_{s}=\frac{\partial}{\partial x_{s}}$.

The Bernstein basis of $\mathbb{P}_{n}$ (space of polynomials of total degree at most $n$ ) and the Bernstein operator are defined respectively by:

$$
b_{\mathbf{i}}^{(n)}(\mathbf{x})=\frac{n !}{\mathbf{i} !} \mathbf{x}^{\mathbf{i}} \quad \text { for } \mathbf{i} \in \Sigma_{n}, \quad B_{n} f(\mathbf{x})=\sum_{\mathbf{i} \in \Sigma_{n}} f\left(\frac{\mathbf{i}}{n}\right) b_{\mathbf{i}}^{(n)}(\mathbf{x})
$$

As $\sum_{\mathbf{i} \in \Sigma_{n}} b_{\mathbf{i}}^{(n)}(\mathbf{x})=1$ and $\sum_{\mathbf{i} \in \Sigma_{n}} \frac{i_{s}}{n} b_{\mathbf{i}}^{(n)}(\mathbf{x})=\mathbf{x}^{\varepsilon_{s}}=x_{s}$, for $1 \leq s \leq d$, where $\varepsilon_{s}=(0,0, \ldots, 1, \ldots, 0)$, then $B_{n}$ is exact on $\mathbb{P}_{1}$.

Let $\left.l_{\mathbf{i}}^{n}, \mathbf{i} \in \boldsymbol{\Sigma}_{\mathbf{n}}\right\}$ be the Lagrange basis of $\mathbb{P}_{n}$ associated with the data points $X_{n}$. Then $l_{\mathbf{i}}^{n}\left(\frac{\mathbf{j}}{n}\right)=\delta_{\mathbf{i j}}$ implies $B_{n} l_{\mathbf{i}}^{n}=b_{\mathbf{i}}^{(n)}$, hence $B_{n}$ is an isomorphism of $\mathbb{P}_{n}$. For $f \in C^{2}(S)$, we have the Voronovskaja type result ([47][79][85]),

$$
\lim n\left[B_{n} f-f\right]=\frac{1}{2} \overline{\mathcal{D}} f
$$

where $\mathcal{D} f$ is the differential operator

$$
\overline{\mathcal{D}} f(x)=\sum_{i<j} x_{i} x_{j}\left(\partial_{i}-\partial_{j}\right)^{2} .
$$


$B_{n}$ and its inverse $A_{n}=B_{n}^{-1}$ in $\mathbb{P}_{n}$ can be expressed as linear differential operators

$$
B_{n}=\sum_{\mathbf{i} \in \boldsymbol{\Sigma}_{\mathbf{n}}} \beta_{\mathbf{i}}^{(n)} D^{\mathbf{i}}, \quad A_{n}=\sum_{\mathbf{i} \in \boldsymbol{\Sigma}_{\mathbf{n}}} \alpha_{\mathbf{i}}^{(n)} D^{\mathbf{i}}
$$

whose coefficients can be computed by recursion. For $0 \leq k \leq n$, define partial inverses

$$
A_{n}^{(k)}=\sum_{\mathbf{i} \in \boldsymbol{\Sigma}_{\mathbf{k}}} \alpha_{\mathbf{i}}^{(n)} D^{\mathbf{i}}
$$

As in section 2.2 for univariate QIs, we can consider the two families of operators: left Bernstein quasi-interpolants (LBQIs) $B_{n}^{(k)}=A_{n}^{(k)} \circ B_{n}$, and right Bernstein quasi-interpolant (RBQIs) $B_{n}^{[k]}=B_{n} \circ A_{n}^{(k)}$, where $B_{n}^{(0)}=B_{n}^{[0]}=B_{n}=$ and $B_{n}^{(n)}=B_{n}^{[n]}=\mathcal{L}_{n}=$ Lagrange interpolation on $X_{n}$.

We have proved [65] that $\left\|B_{n}^{(2)}\right\|_{\infty} \leq 2 d+1$ for all $n \geq 2$, and we conjecture that for all $k \geq 0$, there exists a constant $C_{k}(d)$ such that for all $n \geq k$,

$$
\left\|B_{n}^{(k)}\right\|_{\infty} \leq C_{k}(d) \text {. }
$$

We also conjecture the Voronovskaja-type results

$$
\lim n^{r+1}\left(B_{n}^{(2 r)} f-f\right)=\mathcal{A}_{2 r} f, \lim n^{r+1}\left(B_{n}^{(2 r+1)} f-f\right)=\mathcal{A}_{2 r+1}^{*} f,
$$

where $\mathcal{A}_{2 r}$ and $\mathcal{A}_{2 r+1}^{*}$ are linear differential operators, and the asymptotic expansions

$$
B_{n}^{(2 r)} f \text { and } B_{n}^{(2 r+1)} f \sim f+\frac{c_{r+1}}{n^{r+1}}+\frac{c_{r+2}}{n^{r+2}} \ldots
$$

\subsection{Durrmeyer-Jacobi QIs on a simplex}

One can introduce a Jacobi weight on the simplex in the scalar product of $L_{w}^{2}(S)$ : $w_{\alpha}(x)=x^{\alpha}, \quad\langle f, g\rangle=\int_{S} w_{\alpha}(x) f(x) g(x) d x$, and define the Durrmeyer-Jacobi quasi-interpolants (DJQIs)

$$
M_{n} f=\sum_{i \in \Sigma_{n}} \frac{\left\langle f, b_{i}^{(n)}\right\rangle}{\left\langle e_{0}, b_{i}^{(n)}\right\rangle} b_{i}^{(n)} .
$$

Its eigenvectors are the Jacobi polynomials on the simplex. There holds a Voronovskaja type result [13][79]

$$
\lim n\left(M_{n} f(x)-f(x)\right)=\mathcal{D}_{\alpha} f(x)
$$

where the differential operator $\mathcal{D}_{\alpha}$ is defined by

$$
\mathcal{D}_{\alpha}=x^{-\alpha} \sum_{i<j}\left(\partial_{i}-\partial_{j}\right) x_{i} x_{j}^{\alpha}\left(\partial_{i}-\partial_{j}\right) .
$$


As $M_{n}$ is an isomorphism of $\mathbb{P}_{n}$, one can expand $M_{n}=\sum_{k=0}^{n} \sum_{\mathbf{i} \in \boldsymbol{\Sigma}_{\mathbf{k}}} \beta_{\mathbf{i}}^{(n)} D^{\mathbf{i}}$ and $L_{n}=M_{n}^{-1}=\sum_{k=0}^{n} \sum_{\mathbf{i} \in \boldsymbol{\Sigma}_{\mathbf{n}}} \alpha_{\mathbf{i}}^{(n)} D^{\mathbf{i}}$. As in the univariate case [71], the polynomials $\beta_{\mathbf{i}}^{(n)}$ and $\alpha_{\mathbf{i}}^{(n)}$ are probably linear combinations of Jacobi polynomials on $S([26])$ Setting $L_{n}^{(r)}=\sum_{k=0}^{r} \sum_{\mathbf{i} \in \boldsymbol{\Sigma}_{\mathbf{n}}} \alpha_{\mathbf{i}}^{(n)} D^{\mathbf{i}}$, one can define the left DJQIs $M_{n}^{(r)}=L_{n}^{(r)}$ 。 $M_{n}$, and the right DJQIs $M_{n}^{[r]}=M_{n} \circ L_{n}^{(r)}$, with $M_{n}^{(0)}=M_{n}$ and $M_{n}^{(n)}=P_{n}=$ orthogonal projector on $\mathbb{P}_{n}$ in $L^{2}(S)$. They have the same properties as univariate QIs, and it would be interesting to have detailed proofs, those of [65][66] being only sketched. However, the author thinks that the following operators are still more attractive.

\subsection{Jetter-Stöckler operators on a triangle}

For the sake of simplicity, we describe them over a triangle (with barycentric coordinates $\left.\left\{\lambda_{1}, \lambda_{2}, \lambda_{3}\right\}\right)$ in the case of the Legendre weight $(w=1$, see [42] for the general study on a simplex with Jacobi weight). Using the following notations:

$$
\begin{gathered}
D_{i j}=\partial_{j}-\partial_{i}, i<j, \quad D=\left\{D_{12}, D_{13}, D_{23}\right\}, \Lambda=\left\{\lambda_{1} \lambda_{2}, \lambda_{1} \lambda_{3}, \lambda_{2} \lambda_{3}\right\}, \\
\mathbf{k}=\left(k_{12}, k_{13}, k_{23}\right) \in \mathbb{N}^{3}, \mathcal{D}^{\mathbf{k}}=D_{12}^{k_{12}} D_{13}^{k_{13}} D_{23}^{k_{23}}, \\
\Lambda^{\mathbf{k}}=\left(\lambda_{1} \lambda_{2}\right)^{k_{12}}\left(\lambda_{1} \lambda_{3}\right)^{k_{13}}\left(\lambda_{2} \lambda_{3}\right)^{k_{23}},
\end{gathered}
$$

the authors define the following basic differential operators:

$$
U_{\mathbf{k}}=\frac{1}{\mathbf{k} !}(-1)^{|\mathbf{k}|} \mathcal{D}^{\mathbf{k}} \Lambda^{\mathbf{k}} \mathcal{D}^{\mathbf{k}}, \quad \mathcal{U}_{\ell}=\frac{1}{\ell !} \sum_{|\mathbf{k}|=\ell} U_{\mathbf{k}}, \quad \mathcal{Y}_{n}=\sum_{\ell=0}^{n}\left(C_{n}^{\ell}\right)^{-1} \mathcal{U}_{\ell}
$$

Let $M_{n}$ be the Durrmeyer operator, then they prove that $U_{\mathbf{k}}$ commute with $M_{n}$ for all pairs $(\mathbf{k}, n)$ and that $\mathcal{Y}_{n}$ is the inverse of $M_{n}$ in the space of polynomials $\mathbb{P}_{n}$. Now, for $0 \leq r \leq n$ fixed, they define partial inverses and left Jetter-Stöckler quasi-interpolants (LJSQIs)

$$
\mathcal{Y}_{n}^{(r)}=\sum_{\ell=0}^{r}\left(C_{n}^{\ell}\right)^{-1} \mathcal{U}_{\ell}, \quad M_{n}^{(r)}=\mathcal{Y}_{n}^{(r)} M_{n}
$$

One can also define right JSQIs $M_{n}^{[r]}=M_{n} \mathcal{Y}_{n}^{(r)}$. Both operators $M_{n}^{(r)}$ and $M_{n}^{[r]}$ are exact on $\mathbb{P}_{r}$. Moreover, for $r$ fixed, the left JSQIs have uniformly bounded infinite norms w.r.t. $n$. Finally, the authors prove Voronovskaja-type results:

$$
\lim _{n \rightarrow \infty} C_{n}^{r}\left(f-M_{n}^{(r-1)} f\right)=\mathcal{U}_{r} f
$$




\subsection{Extrapolation}

All operators $\mathcal{B}_{n}$ described in this section have asymptotic expansions of type

$$
\mathcal{B}_{n} f(x) \approx f(x)+\sum_{k \geq r} \frac{\varphi_{k}^{(n)}(f, x)}{n^{k}}
$$

(see e.g. [47] and [85]). In particular, the latter reports interesting numerical results on Richardson extrapolation of classical Bernstein operators on the triangle. It would be interesting to compare these results with those which could be obtained by extrapolating the above QIs.

PARTITIONS-

\section{Univariate spline QIs on uniform partitions}

\subsection{Univariate differential and discrete QIs}

For the construction of QIs with optimal approximation order, we refer to [15] and [16], where general solutions are given, thus completing the initial work by Schoenberg in [80].

\subsection{Near-best spline dQIs}

Consider the family of spline dQIs of order $2 m$ depending on $n+1$ arbitrary parameters $a=\left(a_{0}, a_{1}, \ldots, a_{n}\right), n \geq m$ :

$$
Q_{a} f=\sum_{i \in \mathbb{Z}} \Lambda f(i) M_{2 m}(x-i)
$$

with coefficient functionals

$$
\Lambda f(i)=a_{0} f(i)+\sum_{j=1}^{n} a_{j}(f(i+j)+f(i-j)) .
$$

Setting $\nu(a)=\left|a_{0}\right|+\sum_{j=1}^{n}\left|a_{j}\right|$, then we have $\left\|Q_{a}\right\|_{\infty} \leq \nu(a)$. By imposing that $Q_{a}$ be exact on $\mathbb{P}_{r}$, with $0 \leq r \leq 2 m-1$, we obtain a set of linear constraints: $a \in V_{r} \subset \mathbb{R}^{n+1}$. We say that $Q^{*}=Q_{a^{*}}$ is a near best $d Q I$ if

$$
\nu\left(a^{*}\right)=\min \left\{\nu(a) ; a \in V_{r}\right\} .
$$

There is existence, but in general not unicity, of solutions.

Example: cubic splines (see [40]). There is a unique optimal solution for $n \geq 2$ :

$$
a_{0}^{*}=1+\frac{1}{3 n^{2}}, \quad a_{n}^{*}=-\frac{1}{6 n^{2}}, \quad a_{j}^{*}=0 \text { for } 1 \leq j \leq n-1
$$

Moreover, for all $n \geq 4,\left\|Q^{*}\right\|_{\infty} \leq 1+\frac{2}{3 n^{2}}$. Here are the first values of $\left\|Q^{*}\right\|_{\infty}$ and $\nu\left(a^{*}\right) ; n=1: 1.222 \& 1.666 ; n=2: 1.139 \& 1.166 ; n=3: 1.074 \& 1.074$.

PARTITIONS- 


\subsection{Near-best spline iQIs}

A similar study can be done for integral spline QIs. We refer to [2][40] and we only give an example given in these papers. Setting $a=\left(a_{0}, a_{1}, \ldots, a_{n}\right), n \geq m$ and $M_{i}(x)=M_{2 m}(x-i)$, we consider $Q_{a} f=\sum_{i \in \mathbb{Z}} \Lambda f(i) M_{i}$ with coefficient functionals

$$
\Lambda f(i)=a_{0}\left\langle f, M_{i}\right\rangle+\sum_{j=1}^{n} a_{j}\left(\left\langle f, M_{i-j}\right\rangle+\left\langle f, M_{i+j}\right\rangle\right) .
$$

As in section 4.2, we have $\left\|Q_{a}\right\|_{\infty} \leq \nu(a)$ and we say that $Q^{*}=Q_{a^{*}}$ is a near best $i Q I$ if $\nu\left(a^{*}\right)=\min \left\{\nu(a) ; a \in V_{r}\right\}$. There is existence, but in general not unicity, of solutions.

Example: cubic splines (see [40]). There is a unique optimal solution for $n \geq 2$ :

$$
a_{0}^{*}=1+\frac{2}{3 n^{2}}, \quad a_{n}^{*}=-\frac{1}{3 n^{2}}, \quad a_{j}^{*}=0 \text { for } 1 \leq j \leq n-1
$$

Moreover, for all $n \geq 4,\left\|Q^{*}\right\|_{\infty} \leq 1+\frac{4}{3 n^{2}}$. Here are the first values of $\left\|Q^{*}\right\|_{\infty}$ and $\nu\left(a^{*}\right) ; n=1: 1.5278 \& 2.333 ; n=2: 1.2778 \& 1.333 ; n=3: 1.1481 \& 1.1482$.

\section{Bivariate spline dQIs on uniform partitions}

\subsection{A general construction of dQIs}

Let $\varphi$ be any kind of bivariate B-spline on one of the two classical three- or fourdirectional meshes of the plane (e.g. box-splines, see [7],[12],[19]). Let $\Sigma=\operatorname{supp}(\varphi)$ and $\Sigma^{*}=\Sigma \cap \mathbb{Z}^{2}$. Let $a$ be the hexagonal (or lozenge=rhombus) sequence formed by the values $\left\{\varphi(i), i \in \Sigma^{*}\right\}$. The associated central difference operator $\mathcal{D}$ is an isomorphism of $\mathbb{P}(\varphi)$, the maximal subspace of "complete " polynomials in the space of splines $\mathcal{S}(\varphi)$ generated by the integer translates of the B-spline $\varphi$ (see [12], [69],[71],[72]). Computing the expansion of $a$ in some basis of the space of hexagonal (or lozenge) sequences amounts to expand $\mathcal{D}$ in some basis of central difference operators. Then, computing the formal inverse $\mathcal{D}^{-1}$ allows to define the dQI

$$
\mathcal{Q} f=\sum_{k \in \mathbb{Z}^{2}} \mathcal{D}^{-1} f(k) \varphi(\cdot-k)
$$

which is exact on $\mathbb{P}(\varphi)$. Let us now give two examples which are detailed in [40].

\subsection{Near-best spline dQIs on a three direction mesh}

Example: let $\varphi$ be the $C^{2}$ quartic box-spline. Let $H_{s}$ be the regular hexagon with edges of length $s \geq 1$, centered at the origin (here $\Sigma=H_{2}$ ) and let $H_{s}^{*}=H_{s} \cap \mathbb{Z}^{2}$. The near-best dQIs have coefficient functionals with supports consisting of the 
center and the 6 vertices of $H_{s}^{*}, s \geq 1$. The coefficients of values of $f$ at those points are respectively $1+\frac{1}{2 s^{2}}$ and $-\frac{1}{12 s^{2}}$, therefore the infinite norm of the optimal dQIs $Q_{s}^{*}$ is bounded above by $\nu_{s}^{*}=1+\frac{1}{s^{2}}$. Here are the first values of $\left\|Q^{*}\right\|_{\infty}$ and $\nu_{s}^{*}$; $n=1: 1.34028 \& 2 ; n=2: 1.22917 \& 1.25 ; n=3: 1.10185 \& 1.111$.

\subsection{Near-best spline dQIs on a four direction mesh}

Example: let $\varphi$ be the $C^{1}$ quadratic box-spline. Let $\Lambda_{s}$ be the lozenge (rhombus) with edges of length $s \geq 1$, centered at the origin, and let $\Lambda_{s}^{*}=\Lambda_{s} \cap \mathbb{Z}^{2}$. The near-best dQIs have coefficient functionals with supports consisting of the center and the 4 vertices of $\Lambda_{s}^{*}, s \geq 1$. The coefficients of values of $f$ at those points are respectively $1+\frac{1}{2 s^{2}}$ and $-\frac{1}{8 s^{2}}$, therefore the infinite norm of the optimal dQIs $Q_{s}^{*}$ is bounded above by $\nu_{s}^{*}=1+\frac{1}{s^{2}}$. Here are the first values of $\left\|Q^{*}\right\|_{\infty}$ and $\nu_{s}^{*}$; $n=1: 1.5 \& 2 ; n=2: 1.25 \& 1.25 ; n=3: 1.111 \& 1.111$.

\section{Univariate spline QIs on non uniform partitions}

\subsection{Uniformly bounded dQIs}

Let us only give an example: we start from a family of DQIs of degree $m$ which are exact on $\mathbb{P}_{2}$.

$$
Q_{2} f=\sum_{j \in J} \lambda_{j}^{(2)}(f) B_{j}, \quad \lambda_{j}^{(2)}(f)=f\left(\theta_{j}\right)-\frac{1}{2}\left(\theta_{j}^{2}-\theta_{j}^{(2)}\right) D^{2} f\left(\theta_{j}\right) .
$$

We recall the expansion [52][53]

$$
A_{j}^{(2)}=\theta_{j}^{2}-\theta_{j}^{(2)}=\frac{1}{(m-1)^{2}(m-2)} \sum_{(r, s) \in E_{m}^{2}, r \neq s}\left(t_{j+r}-t_{j+s}\right)^{2}>0 .
$$

On the other hand, $\frac{1}{2} D^{2} f\left(\theta_{j}\right)$ can be replaced on the space $\mathbb{P}_{2}$ by the second order divided difference $\left[\theta_{j-1}, \theta_{j}, \theta_{j+1}\right] f$, therefore the dQI defined by

$$
Q_{2}^{*} f=\sum_{j \in J} \mu_{j}^{(2)}(f) B_{j}, \quad \mu_{j}^{(2)}(f)=f\left(\theta_{j}\right)-A_{j}^{(2)}\left[\theta_{j-1}, \theta_{j}, \theta_{j+1}\right] f,
$$

is also exact on $\mathbb{P}_{2}$. Moreover, one can write

$$
\mu_{i}^{(2)}(f)=a_{i} f_{i-1}+b_{i} f_{i}+c_{i} f_{i+1}
$$

with $a_{i}=-A_{i}^{(2)} / \Delta \theta_{i-1}\left(\Delta \theta_{i-1}+\Delta \theta_{i}\right), \quad c_{i}=-A_{i}^{(2)} / \Delta \theta_{i}\left(\Delta \theta_{i-1}+\Delta \theta_{i}\right)$, and $b_{i}=1+A_{i}^{(2)} / \Delta \theta_{i-1} \Delta \theta_{i}$, So, according to the introduction

$$
\left\|Q_{2}^{*}\right\|_{\infty} \leq \max _{i \in J}\left(\left|a_{i}\right|+\left|b_{i}\right|+\left|c_{i}\right|\right) \leq 1+2 \max _{i \in J} \frac{A_{i}^{(2)}}{\Delta \theta_{i-1} \Delta \theta_{i}} .
$$

The following theorem [4] extends a result given for quadratic splines in [4][73][75]. 
Theorem 1. For any degree $m$, the $d Q I s Q_{2}^{*}$ are UB. More specifically, for all partitions of $I$ :

$$
\left\|Q_{2}^{*}\right\|_{\infty} \leq\left[\frac{1}{2}(m+4)\right]
$$

\subsection{Uniformly bounded iQIs}

General types of integral QIs are studied in [21][63][68]. Here, we have chosen to study a family of QIs that we call Goodman-Sharma type iQIs, as they first appear in [38]. They seem simpler and more interesting than those we have studied in [68]. The simpler GS-type IQI can be written as follows

$$
G_{1} f=f\left(t_{0}\right) B_{0}+\sum_{i=1}^{n+m-2} \tilde{\mu}_{i}(f) B_{i}+f\left(t_{n}\right) B_{n+m-1},
$$

where the integral coefficient functionals are defined by

$$
\tilde{\mu}_{i}(f)=\int_{0}^{1} \tilde{M}_{i-1}(t) f(t) d t
$$

$\tilde{M}_{i-1}(t)$ being the B-spline of degree $m-2$ with support $\tilde{\Sigma}_{i-1}=\left[t_{i-m+1}, t_{i}\right]$, normalized by $\tilde{\mu}_{i}^{(0)}=\tilde{\mu}_{i}\left(e_{0}\right)=\int_{0}^{1} \tilde{M}_{i-1}(t)=1$. It is easy to verify that $G_{1}$ is exact on $\mathbb{P}_{1}$ and that $\left\|G_{1}\right\|_{\infty}=1$. We shall study the family of GS-type iQIs defined by

$$
G_{2} f=f\left(t_{0}\right) B_{0}+\sum_{i=1}^{n+m-2}\left[a_{i} \tilde{\mu}_{i-1}(f)+b_{i} \tilde{\mu}_{i}(f)+c_{i} \tilde{\mu}_{i+1}(f)\right] B_{i}+f\left(t_{n}\right) B_{n+m-1},
$$

which are exact on $\mathbb{P}_{2}$. The three constraints $G_{2} e_{k}=e_{k}, \quad k=0,1,2$, lead to the following system of equations, for $1 \leq i \leq n+m-2$ :

$$
a_{i}+b_{i}+c_{i}=1, \quad \theta_{i-1} a_{i}+\theta_{i} b_{i}+\theta_{i+1} c_{i}=\theta_{i}, \quad \tilde{\mu}_{i-1}^{(2)} a_{i}+\tilde{\mu}_{i}^{(2)} b_{i}+\tilde{\mu}_{i+1}^{(2)} c_{i}=\theta_{i}^{(2)} .
$$

This is a consequence of the following facts

$$
\begin{gathered}
\tilde{\mu}_{i}\left(e_{1}\right)=\int_{0}^{1} t \tilde{M}_{i-1}(t) d t=\frac{1}{m} \sum_{s=1}^{m} t_{i-m+s}=\theta_{i}, \\
\tilde{\mu}_{i}^{(2)}=\mu_{i}\left(e_{2}\right)=\int_{0}^{1} t^{2} \tilde{M}_{i-1}(t) d t=\frac{2}{m(m+1)} \tilde{s}_{2}\left(T_{i}\right) \\
=\frac{2}{m(m+1)} \sum_{1 \leq r \leq s \leq m} t_{i-m+r} t_{i-m+s}
\end{gathered}
$$


Theorem 2. For any degree $m$, the $i Q I_{s} G_{2}$ are UB. More specifically, for all partitions of $I$ :

$$
\left\|G_{2}\right\|_{\infty} \leq 5
$$

The detailed proof will be given in [78].

\subsection{Near-best dQIs}

Let us consider the family of dQIs of degree $m$ defined, for the sake of simplicity, on $I=\mathbb{R}$ endowed with an arbitrary non-uniform increasing sequence of knots $T=\left\{t_{i} ; i \in \mathbb{Z}\right\}$, by

$$
Q f=Q_{p, q} f=\sum_{i \in \mathbb{Z}} \mu_{i}(f) B_{i}
$$

Their coefficient functionals depend on $2 p+1$ parameters, with $p \geq m$ :

$$
\mu_{i}(f)=\sum_{s=-p}^{p} \lambda_{i}(s) f\left(\theta_{i+s}\right)
$$

and they are exact on the space $\mathbb{P}_{q}$, where $q \leq \min (m, 2 p)$. The latter condition is equivalent to $Q e_{r}=e_{r}$ for all monomials of degrees $0 \leq r \leq q$. It implies that for all indices $i$, the parameters $\lambda_{i}(s)$ satisfy the system of $q+1$ linear equations:

$$
\sum_{s=-p}^{p} \lambda_{i}(s) \theta_{i+s}^{r}=\theta_{i}^{(r)}, \quad 0 \leq r \leq q .
$$

The matrix $V_{i} \in \mathbb{R}^{(q+1) \times(2 p+1)}$ of this system, with coefficients $V_{i}(r, s)=\theta_{i+s}^{r}$, is a Vandermonde matrix of maximal rank $q+1$, therefore there are $2 p-q$ free parameters. Denoting $b_{i} \in \mathbb{R}^{q+1}$ the vector in the right hand side, with components $b_{i}(r)=\theta_{i}^{(r)}, \quad 0 \leq r \leq q$, we consider the sequence of minimization problems, for $i \in \mathbb{Z}$ :

$$
\min \left\|\lambda_{i}\right\|_{1}, \quad V_{i} \lambda_{i}=b_{i} .
$$

We have seen in the introduction that $\nu_{1}^{*}(Q)=\max _{i \in \mathbb{Z}} \min \left\|\lambda_{i}\right\|_{1}$ is an upper bound of $\left\|Q_{q}\right\|_{\infty}$ which is easier to evaluate than the true norm of the dQI.

Theorem 3. The above minimization problems have always solutions, which, in general, are non unique.

The objective function being convex and the domains being affine subspaces, these classical optimization problems have always solutions, in general non unique.

Example of optimal dQIs are given in [1][4][40]. 


\section{Bivariate quadratic spline dQIs on non uniform criss-cross triangulations}

At the author's knowledge, the only bivariate box-splines which have been extended to non uniform partitions of the plane are $C^{1}$-quadratic box-splines on criss-cross triangulations [20][62]. Recently, we have constructed a set of B-splines generating the space of quadratic splines on a rectangular domain and we have defined a discrete quasi-interpolant which is exact on $\mathbb{P}_{2}$ and uniformly bounded independently of the partition [74]-[76].

\section{Abbreviations for publishers and journals}

Publishers: AP=Academic Press, New-York ; BAS=Bulgarian Academy of Science, Sofia; BV=Birkhäuser-Verlag, Basel; CUP=Cambridge University Press; JWS=John Wiley \& Sons, New-York; K=Kluwer, Dordrecht; NH= North-Holland, Amsterdam; NP=Nashboro Press, Brentwood; SV=Springer-Verlag, Berlin; SIAM=Society for Industrial and Applied Mathematics, Philadelphia; VUP=Vanderbilt University Press, Nashville.

Journals: $\mathrm{AiCM}=$ Advances in Comput. Mathematics; ATA=Approximation Theory and its Applications (now Analysis in Theory and Applications);

$\mathrm{CAGD}=$ Computer Aided Geometric Design; JAT=Journal of Approximation Theory; JCAM=Journal of Computational and Applied Mathematics.

Proceedings: AT2=Approximation Theory II, G.G. Lorentz, C.K. Chui, L.L. Schumaker (eds), AP 1976; AT4 \& AT5=Approximation Theory IV \& V, C.K. Chui, L.L. Schumaker, J.D. Ward (eds), AP 1983 and 1986; CMSB=Colloquia Mathematica Soc. Janos Bolyai; CS02=Curve and Surface Fitting (St Malo 2002), A. Cohen, J.L. Merrien and L.L. Schumaker (eds), NP 2003.

Preprints: PI=Prépublications IRMAR, Inst. de Recherche Math. de Rennes.

\section{References}

[1] D. Barrera, M.J. Ibanẽz, P. Sablonnière: Near-best quasi-interpolants on uniform and nonuniform partitions in one and two dimensions. In CS02 (2003), $31-40$.

[2] D. Barrera, M.J. Ibanẽz, P. Sablonnière, D. Sbibih: Near-minimally normed spline quasi-interpolants on uniform partitions. PI 04-12, 2004. (Submitted).

[3] D. Barrera, M.J. Ibanẽz, P. Sablonnière, D. Sbibih: Near-best quasiinterpolants associated with $\mathrm{H}$-splines on a three directional mesh. PI 04-14, 2004 (Submitted). 
[4] D. Barrera, M.J. Ibanẽz, P. Sablonnière, D. Sbibih: Near-best univariate spline discrete quasi-interpolants on non-uniform partitions. PI 04-15, 2004 (Submitted).

[5] H. Berens, H.J. Schmidt and Y. Xu: Bernstein-Durrmeyer polynomials on a simplex. JAT 68 (1992), 247-261.

[6] H. Berens and Xu: On Bernstein-Durrmeyer polynomials with Jacobi weights. In Approx. Theory and Functional Anal., C.K. Chui (ed.), AP (1991), 25-46.

[7] B.D. Bojanov, H.A. Hakopian, A.A. Sahakian: Spline functions and multivariate interpolation, K 1993.

[8] C. de Boor: Splines as linear combinations of B-splines. In AT2 (1976), 1-47.

[9] C. de Boor: Quasi-interpolants and approximation power of multivariate splines. in Computation of Curves and Surfaces, W. Dahmen, M. Gasca and C.A. Micchelli (eds), K (1990), 313-345.

[10] C. de Boor: A practical guide to splines, SV 2001. (revised edition).

[11] C. de Boor and M.G. Fix: Spline approximation by quasi-interpolants. JAT 8 (1973), 19-54.

[12] C. de Boor, K. Höllig and S. Riemenschneider: Box-splines. SV 1992.

[13] D. Braess and Ch. Schwab: Approximation on simplices with respect to weighted Sobolev norms. JAT 103 (2000), 329-337.

[14] C. Brezinski, M. Redivo-Zaglia: Extrapolation methods, theory and practice, NH 1992.

[15] P.L. Butzer, M. Schmidt, E.L. Stark, L. Vogt: Central factorial numbers, their main properties and some applications. Numer. Funct. Anal. and Optimiz. 10 (1989), 419-488.

[16] P.L. Butzer, M. Schmidt: Central factorial numbers and their role in finite difference calculus and approximation. In Approximation theory, J. Szabados and K. Tandori (eds), CMSB 58, NH(1990), 127-150.

[17] G. Chen, C.K. Chui, M.J. Lai: Construction of real-time spline quasiinterpolation schemes, ATA 4 (1988), 61-75.

[18] W. Chen and Z. Ditzian: Multivariate Durrmeyer-Bernstein operators, in Israel mathematical conference proceedings, Vol. 4, Conference in honour of A. Jakimowski (1991), 109-119.

[19] C.K. Chui : Multivariate splines. SIAM 1992. 
[20] Chui, Schumaker, Wang: Concerning $C^{1} \mathrm{~B}$-splines on triangulations of nonuniform rectangular partitions. ATA 1 (1984), 11-18.

[21] Z. Ciesielski: Local spline approximation and nonparametric density estimation. In Constructive theory of functions '87, BAS (1988), 79-84.

[22] F. Costabile, M.I. Gualtieri, S. Serra, Asymptotic expansions and extrapolation for Bernstein polynomials with applications. BIT 36 (1996), 676-687.

[23] C. Dagnino, P. Lamberti: Numerical integration of 2D integrals based on local bivariate $C^{1}$ quasi-interpolating splines. Adv. Comput. Math. 8 (1998), 19-31.

[24] M.M. Derriennic: Sur l'approximation des fonctions intégrables sur $[0,1]$ par des polynômes de Bernstein modifiés. JAT 31, No 4 (1981), 325-343.

[25] M.M. Derriennic: Polynômes de Bernstein modifiés sur un simplexe T de $\mathbb{R}^{l}$. Problème des moments. In Polynômes orthogonaux et applications, C. Brezinski et al. (eds), LNM 1171, SV (1985), 296-301.

[26] M.M. Derriennic: Polynômes orthogonaux de type Jacobi sur un triangle. C.R. Acad. Sci. Paris Ser I, 300 (1985), 471-474.

[27] M.M. Derriennic: On multivariate approximation by Bernstein type polynomials. JAT 45 (1985), 155-166.

[28] M.M. Derriennic: Linear combinations of derivatives of Bernstein type polynomials on a simplex. CMSB 58 (1990), 197-220.

[29] R.A. DeVore, G.G. Lorentz: Constructive Approximation. SV 1993.

[30] A.T. Diallo: Rate of convergence of Bernstein quasi-interpolants. Publ. IC/95/295, Int. Centre for Theoretical Physics, Miramare-Trieste, 1995.

[31] Z. Ditzian: Multidimensional Jacobi type Bernstein-Durrmeyer operators. Acta Sci. Math. (Szeged) 60 (1995), 225-243.

[32] Z. Ditzian, V. Totik: Moduli of smoothness. SV 1987.

[33] J.L. Durrmeyer: Une formule d'inversion de la transformée de Laplace: applications à la théorie des moments. Thèse, Université de Paris, 1967.

[34] H.H. Gonska, J. Meier: Quantitative theorems on approximation by BernsteinStancu operators. Calcolo 21(1984), 317-335.

[35] H.H. Gonska, J. Meier: A bibliography on approximation of functions by Bernstein type operators, in AT4, AP (1983), 739-785.

[36] H.H. Gonska, J. Meier: A bibliography on approximation of functions by Bernstein type operators. (suppl. 1986), in AT5, AP (1986), 621-654. 
[37] T.N.T. Goodman, H. Oruç, G.M. Phillips: Convexity and generalized Bernstein polynomials. Proc. of the Edinburgh Math. Soc. 42 (1999), 179-190.

[38] T.N.T. Goodman and A. Sharma: A modified Bernstein-Schoenberg operator. In Constructive theory of functions '87, BAS (1988), 166-173.

[39] T.N.T. Goodman and A. Sharma: A Bernstein type operator on the simplex. Mathem. Balkanica 5 (1991), 129-145.

[40] M.J. Ibañez-Pérez: Cuasi-interpolantes spline discretos con norma casi minima : teoria y aplicaciones. Tesis, Univ.de Granada (Sept. 2003).

[41] K. Jetter, J. Stöckler: An identity for multivariate Bernstein polynomials, CAGD 20(2003), 563-577.

[42] K. Jetter, J. Stöckler: New polynomial preserving operators on simplices. Report Nr 242, University of Dortmund, 2003 (Submitted).

[43] V. Kac, P. Cheung: Quantum calculus, SV, New-York, 2002.

[44] Y. Kageyama: Generalization of the left Bernstein quasi-interpolants. JAT 94, No 2 (1998), 306-329.

[45] Y. Kageyama: A new class of modified Bernstein operators. JAT 101, No 1 (1999), 121-147.

[46] M.J. Lai: On dual functionals of polynomials in B-form, JAT 67, No 1 (1991), 19-37.

[47] M.J. Lai: Asymptotic formulae of multivariate Bernstein approximation, JAT 70 No 2 (1992), 229-242.

[48] B.G. Lee, T. Lyche, L.L. Schumaker: Some examples of quasi-interpolants constructed from local spline projectors. In Mathematical methods for curves and surfaces: Oslo 2000, T. Lyche and L.L. Schumaker (eds), VUP (2001), 243-252.

[49] G.G. Lorentz: Bernstein polynomials. University of Toronto Press, 1953.

[50] T. Lyche and L.L. Schumaker: Local spline approximation methods. JAT 15 (1975), 294-325.

[51] P. Mache and D.H. Mache: Approximation by Bernstein quasi-interpolants. Numer. Funct. Anal. and Optimiz. 22, 1\& 2 (2001), 159-175.

[52] J.M. Marsden, I.J. Schoenberg: On variation diminishing spline approximation methods. Mathematica (Cluj) 31 (1966), 61-82.

[53] J.M. Marsden, I.J. Schoenberg: An identity for spline functions with applications to variation diminishing spline approximation. JAT 3 (1970), 7-49. 
[54] J.M. Marsden: Operator norm bounds and error bounds for quadratic spline interpolation, In: Approximation Theory, Banach Center Publications, vol. 4 (1979), 159-175.

[55] E. Neuman: Moments and Fourier transforms of B-splines. JCAM 7, 51-62.

[56] H. Oruç and G.M. Phillips: q-Bernstein polynomials and Bézier curves. JCAM 151 (2003), 1-12.

[57] G. M. Phillips: On generalized Bernstein polynomials. In Numerical Analysis: A.R. Mitchell 75-th birthday Volume, D.F. Griffiths and G.A. Watson (eds), World Scientific, Singapore (1996), 263-269.

[58] G. M. Phillips: Bernstein polynomials based on the q-integers. Annals of Numer. Math. 4 (1997), 511-518.

[59] G. M. Phillips: Interpolation and approximation by polynomials. SV 2003.

[60] M.J.D. Powell: Approximation theory and methods. CUP 1981.

[61] P. Sablonnière: Opérateurs de Bernstein-Jacobi. Rapport ANO 37, Université de Lille, 1981 (unpublished).

[62] P. Sablonnière: Bernstein-Bézier methods for the construction of bivariate spline approximants. CAGD 2 (1985), 29-36.

[63] P. Sablonnière: Positive spline operators and orthogonal splines. JAT 52 (1988), 28-42.

[64] P. Sablonnière: Bernstein quasi-interpolants on [0, 1]. In Multivariate Approximation Theory IV, C.K. Chui, W. Schempp and K. Zeller (eds), ISNM, Vol. 90, BV (1989), 287-294.

[65] P. Sablonnière: Bernstein quasi-interpolants on a simplex. Meeting Konstruktive Approximationstheorie, Oberwolfach (July 30-August 5, 1989). Publ. LANS 21, INSA de Rennes, 1989 (unpublished).

[66] P. Sablonnière: Bernstein type quasi-interpolants. In Curves and surfaces, P.J. Laurent, A. Le Méhauté, L.L. Schumaker (eds), AP (1991), 421-426.

[67] P. Sablonnière: A family of Bernstein quasi-interpolants on [0,1]. ATA 8:3 (1992), 62-76.

[68] P. Sablonnière and D. Sbibih: Spline integral operators exact on polynomials. ATA 10:3 (1994), 56-73.

[69] P. Sablonnière: Quasi-interpolants aasociated with H-splines on a threedirection mesh. JCAM 66 (1996), 433-442. 
[70] P. Sablonnière: Representation of quasi-interpolants as differential operators and applications. In New developments in approximation theory, M.W. Müller, M.D. Buhmann, D.H. Mache, M. Felten (eds). ISNM Vol. 132, BV (1999), 233-253.

[71] P. Sablonnière: Quasi-interpolantes sobre particiones uniformes, First meeting in Approximation Theory, Ubeda, Spain, July 2000. PI 00-38, 2000.

[72] P. Sablonnière: H-splines and quasi-interpolants on a three-directional mesh. In Advanced Problems in Constructive Approximation, M.D. Buhmann and D. Mache (eds), ISNM Vol. 142, BV (2002), 187-201.

[73] P. Sablonnière: On some multivariate quadratic spline quasi-interpolants on bounded domains. In Modern Developments in Multivariate Approximation, W. Haussmann et al. (eds), ISNM Vol. 145, BV (2003), 263-278.

[74] P. Sablonnière: BB-coefficients of basic bivariate quadratic splines on rectangular domains with uniform criss-cross triangulations. PI 02-56, 2002.

[75] P. Sablonnière: Quadratic spline quasi-interpolants on bounded domains of $\mathbb{R}^{d}, d=1,2,3$. Spline and radial functions, Rend. Sem. Univ. Pol. Torino, Vol. 61 (2003), 61-78.

[76] P. Sablonnière: BB-coefficients of bivariate quadratic B-splines on rectangular domains with non-uniform criss-cross triangulations. PI 03-14, March 2003.

[77] P. Sablonnière: Refinement equation and subdivision algorithm for quadratic B-splines on non-uniform criss-cross triangulations. Int. Conf. on Wavelets and splines, St Petersburg, 2003 (submitted). PI 03-35, October 2003.

[78] P. Sablonnière: Near-best univariate spline integral quasi-interpolants on nonuniform partitions. PI 2004 (In preparation).

[79] Th. Sauer: The genuine Bernstein-Durrmeyer operator on a simplex. Results Math. 26 (1994), 99-130.

[80] I.J. Schoenberg: Cardinal spline interpolation, CBMS-NSF Regional Conference Series in Applied Mathematics, vol. 12, SIAM, Philadelphia 1973.

[81] L.L. Schumaker: Spline functions: basic theory, JWS 1981.

[82] A. Sidi: Practical extrapolation Methods, CUP 2003.

[83] D.D. Stancu: Approximation properties of a class of linear positive operators. Studia Univ. Babes-Bolyai 15 (1970), 31-38.

[84] G. Vlaic: On the approximation of bivariate functions by the Stancu operator for a triangle. Analele Univ. Timişoara 36, Seria Mat.-Inform.(1998), 149-158. 
[85] G. Walz: Asymptotic expansions for multivariate polynomial approximation. JCAM 122 (2000), 317-328.

[86] Wu Zhengchang: Norm of Bernstein left quasi-interpolant operator. JAT 66 (1991), 36-43.

Centre de Mathématiques

INSA de Rennes

20 Avenue des Buttes de Coësmes, CS 14315

35043-Rennes cedex, France

Email address: psablonn@insa-rennes.fr 